\title{
LETTER
}

\section{The functional genomics of an eco-evolutionary feedback loop: linking gene expression, trait evolution, and community dynamics}

Lutz Becks*, Stephen P. Ellner Laura E. Jones and Nelson G. Hairston Jr. Department of Ecology and Evolutionary Biology, Cornell University, Ithaca, NY 14853, USA

*Correspondence and present address: Max-Planck Institute for Evolutionary Biology, D-24306 Plön, Germany.

E-mail: Ibecks@evolbio.mpg.de

\begin{abstract}
Feedbacks between ecological and evolutionary change may play important roles in community and ecosystem functioning, but a complete eco-evolutionary feedback loop has not been demonstrated at the community level, and we know little about molecular mechanisms underlying this kind of eco-evolutionary dynamics. In predator-prey (rotifer-alga) microcosms, cyclical changes in predator abundance generated fluctuating selection for a heritable prey defence trait, cell clumping. Predator population growth was affected more by prey evolution than by changes in prey abundance, and changes in predator abundance drove further prey evolution, completing the feedback loop. Within a predator-prey cycle, genes up-regulated as clumping declined were down-regulated as clumping increased, and vice-versa. Genes changing most in expression tended to be associated with defence or its cost. Expression patterns of individual genes differed greatly between consecutive cycles (often reversing direction), suggesting that a particular phenotype may be produced by several (perhaps many) different gene transcription pathways.
\end{abstract}

\section{Keywords}

Chemostat dynamics, eco-evolutionary dynamics, eco-evolutionary feedback loop, microarray, microcosm, predator-prey.

Ecology Letters (2012) 15: 492-501

\section{INTRODUCTION}

The goal of defining the mechanistic links between genotypes and phenotypes has increasingly motivated studies in systems biology over the past decade, particularly as methods for quantifying levels of gene expression have been developed and refined (e.g. Gibson 2002). Populations often undergo substantial evolutionary changes in ecologically important traits within a few generations, on the same time scale as the ecological changes that drive them (e.g. Hendry \& Kinnison 1999; Hairston et al. 2005; Ellner et al. 2011). These rapid changes, caused by strong selection, create an opportunity to identify the genes and molecular mechanisms underlying rapid evolutionary responses to changes in the environment.

Many documented examples of rapid evolution involve a single environmental change (e.g. disturbance and invasion of a new habitat) that evokes a one-time response. Over short time-scales, natural selection in the wild often fluctuates (Siepielski et al. 2009), so that the evolutionary response to selection involves recurrent changes that alternate in direction. Recurrent evolutionary change can also occur as a result of eco-evolutionary feedbacks within a population or community (Abrams 2000; Jones \& Ellner 2007). An eco-evolutionary feedback loop occurs when environmental change causes natural selection in a population, and the resulting trait evolution then modifies the environment, causing further selection and evolution, and so on (Fussmann et al. 2007; Post \& Palkovacs 2009; Schoener 2011). The potential for recurrent rapid evolution of traits critical to interspecific interactions lies at the heart of recent suggestions that eco-evolutionary feedback loops may be important in the functioning of communities and ecosystems (Fussmann et al. 2007; Pelletier et al. 2009; Schoener 2011). However, despite this potential importance, recent reviews of eco-evolutionary dynamics have concluded that there is no system in which all of the components of the feedback loop have been demonstrated (Post \& Palkovacs 2009; Schoener 2011), although it could be argued that frequency- and density-dependent selection (Sinervo et al. 2000; Hanski 2011) within a population count.

Herein, we present laboratory experiments demonstrating a complete eco-evolutionary feedback loop that produces recurrent rapid evolution. We identify genes that allowed adaptation to a changing environment by tracking the changes in gene expression that accompanied trait evolution. Because the same pattern of fluctuating selection and response occurred repeatedly, we could also examine whether the relationship between changes at the phenotypic and gene expression levels remains constant across multiple bouts of selection.

Our experiments used aquatic predator-prey microcosms in which the rotifer Brachionus calyciflorus consumes the green alga, Chlamydomonas reinhardtii. Cyclic fluctuations in predator and prey abundance drive, and are in turn driven by, heritable changes in a prey trait (cell clumping) that provides defence against predation. Although Chlamydomonas typically occurs as single cells when grown alone, it instead grows primarily as palmelloid clumps of 2-140 cells after multiple generations in the presence of the rotifers. Although others have found clump formation in Chlamydomonas to be inducible in the presence of Brachionus (Lürling \& Beekman 2006), in our system it is a highly heritable trait, retained even after nine generations in the absence of rotifers, with broad sense heritability (the only calculation possible for clonal lineages) of $H^{2}=0.97$ (Becks et al. 2010). We show below that sufficiently large clumps are protected against rotifer predation, but in the absence of predators, defended phenotypes exhibit significantly slower population growth (Becks et al. 2010). This 
trade-off between defence and competitive ability leads to fluctuating selection as a function of predator abundance and resource availability.

Eco-evolutionary predator-prey dynamics have been explored theoretically (e.g. Levin 1972; Abrams 2000; Jones \& Ellner 2007) and shown empirically to occur in laboratory microcosms with the same predator, but a different alga (Chlorella vulgaris) as prey (Yoshida et al. 2003, 2004). However, it was not possible to track the temporal dynamics of any trait relevant to Brachionus-Chlorella interactions because defended Chlorella cells (which pass undigested through the rotifer gut; Meyer et al. 2006) are morphologically indistinguishable from undefended cells, and no genetic markers for the defence trait are known. In contrast, Chlamydomonas has a visible heritable defence trait (clump formation) that evolves readily in the presence of Bracbionus. This allows us to demonstrate and analyse here a complete eco-evolutionary feedback loop in this system by (1) determining the number of cells per clump necessary to provide effective defence, (2) showing how rotifer growth rate depends on both total cell density and the distribution of clump sizes and (3) calculating the relative contributions of ecological (changes in cell density) and evolutionary (change in cell clumping) processes to a key component of this interaction (variation in rotifer population growth rate).

The sequencing of the Chlamydomonas genome (Merchant et al. 2007) and the availability of functional annotation tools (Thimm et al. 2004; Lopez et al. 2011) let us use functional-genomic methods (microarrays) to determine how gene expression changed during the ecoevolutionary cycles. The suite of genes that changed in concert with the degree of cell clumping included annotated sequences likely involved with both the clumping trait itself (i.e. cell wall and cycle) and with its costs (i.e. nitrogen deprivation). Unexpectedly, completely different suites of genes were up or down-regulated during two sequential predator-prey cycles with very similar phenotypic clumping responses to selection. This suggests that several (possibly many) different gene transcription pathways can produce the same pattern of trait dynamics.

\section{MATERIAL AND METHODS}

\section{Chemostat experiments}

Predator-prey dynamics were followed in four replicate chemostats (continuous-flow aquatic culture vessels) supplied with nitrogenlimited sterile medium that was otherwise sufficient in all nutrient constituents (Fussmann et al. 2000; Yoshida et al. 2003; Becks et al. 2010). Three chemostats contained $380 \mathrm{~mL}$ of medium, whereas the fourth, used for our algal gene expression study, contained $3000 \mathrm{~mL}$ so as to provide sufficient material for genetic analysis. Brachionus calyciflorus was isolated originally from Milwaukee harbour, Wisconsin, and provided by M. Boraas. Chlamydomonas reinhardtii was obtained from the University of Texas algal culture collection (UTEX 89). Both species reproduced asexually in our chemostat system. Although $B$. calyciflorus is naturally cyclically parthenogenetic, the rotifers in our stock culture have evolved not to produce males because the bubbling in our chemostats prevents mating; we used only one mating type of Chlamydomonas. Absence of sexual reproduction does not preclude the processes of microevolution (Bell 2009) that we study, and it is commonly accepted that asexual species can evolve (Lenski et al. 1991; Barrett et al. 2005). We used a Chlamydomonas lineage, founded from our original Chlamydomonas stock and then exposed continuously to
Brachionus predation for 6 months in chemostat culture prior to this study during which Chlamydomonas evolved to form clumps (the 'grazed Chlamydomonas' lineage in Becks et al. 2010).

Chemostats were sampled daily by syringe through stoppers in two ports near the bottom and top of each chemostat; all abundances discussed here are the mean value from the top- and bottom-port samples. Algal cell abundances and clump sizes (cells per clump) were counted at $400 \times$ under a compound microscope using a hemacytometer (Improved Neubauer, Hauser Scientific, PA, USA). Rotifer abundances were enumerated using a stereo-dissecting microscope. Differences in the lengths of chemostat runs (50-115 days) resulted from technical issues such as wall growth or contamination that required runs to be terminated.

We determined the threshold number of algal cells per clump above which Chlamydomonas was defended by calculating the fraction of variance $\left(r^{2}\right)$ in rotifer per-capita growth rate explained by the abundance of 'potentially edible' cells (the total number of algal cells in clumps within the edible size range). We calculated first the $r^{2}$ for rotifer growth rate (at each time, in each chemostat) that could be explained by the abundance of cells occurring singly, which was low. We then determined the fraction of variance explained by the abundance of cells occurring either singly or in clumps of two cells, which was greater. We continued this process for cells occurring either singly, in two-cell clumps or in three-cell clumps, and so on sequentially up to clumps of 16 cells. The result for each chemostat was a hump-shaped relationship that increased up to the threshold number of cells above which clumps were defended. The relationship between $r^{2}$ and the largest clump size included was described by a spline regression fitted in R (R Development Core Team 2009) to the across-replicate chemostat means, using the gam function in mgcv (Wood 2000). We interpret the clump size at which the spline regression curve is largest to be the maximum size of clumps that the rotifers regularly consumed (i.e. that inedibility occurred at a threshold clump size). This analysis assumes a priori that a sharp division exists between edible and inedible clump sizes, so we also considered a gradual decrease in edibility with increasing clump size, represented by a sigmoid curve with fitted location and slope; the best-fitting slope parameter was very high and therefore produced a sharp division between fully edible and completely inedible clumps. For the four chemostat runs separately, the peak in $r^{2}$ occurs at 6-10 cells per clump (Fig. S1).

To quantify cycle periods and phase lags, the data were first smoothed and interpolated to continuous time (e.g. Fig. 1), omitting an initial transient of 14 days prior to the onset of regular cycles. The first peak in the average of the autocorrelation functions for the two species and mean algal clump size was our estimate of the cycle period. The delay at which the cross-correlation between two data series is maximised was our estimate of the time delay between them. Phase lags were estimated by dividing estimated time delay by estimated period.

\section{Molecular dynamics: biological material for expression analysis}

We tracked changes in Chlamydomonas gene expression throughout two complete cycles of algal and rotifer abundance and concurrent cycles in algal defence. Algal samples were taken from one chemostat at intervals of approximately every 10 days (eight dates total), chosen to capture critical transitions in cell clumping (Fig. 1A). A sample taken at the start of the experiment from the culture that was used to 

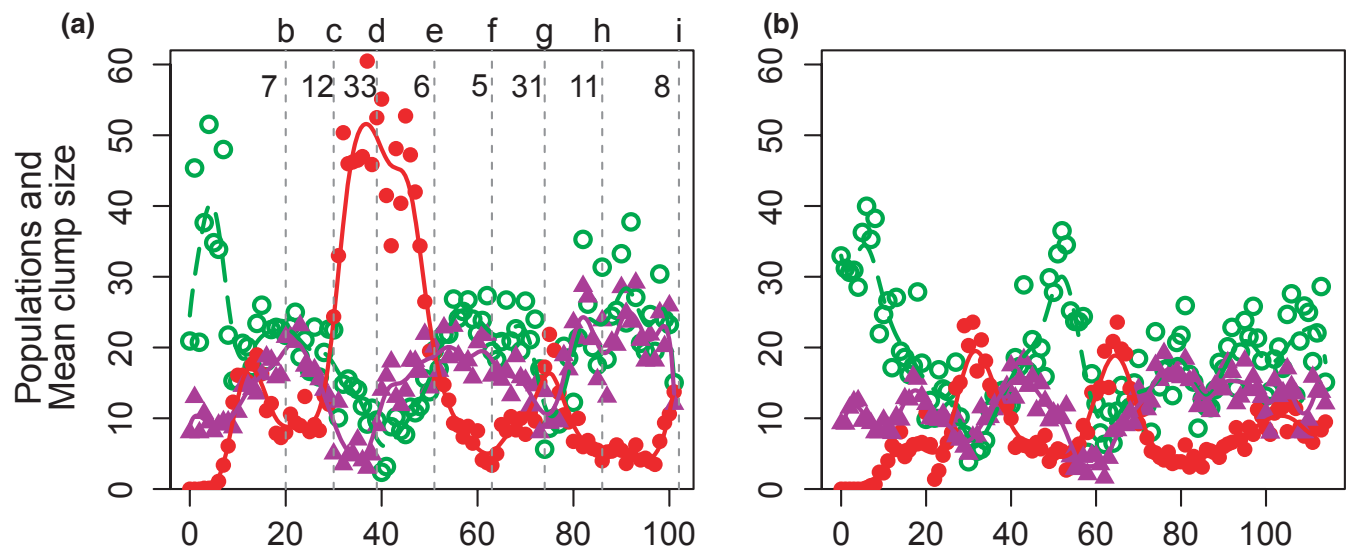

(c)

(d)
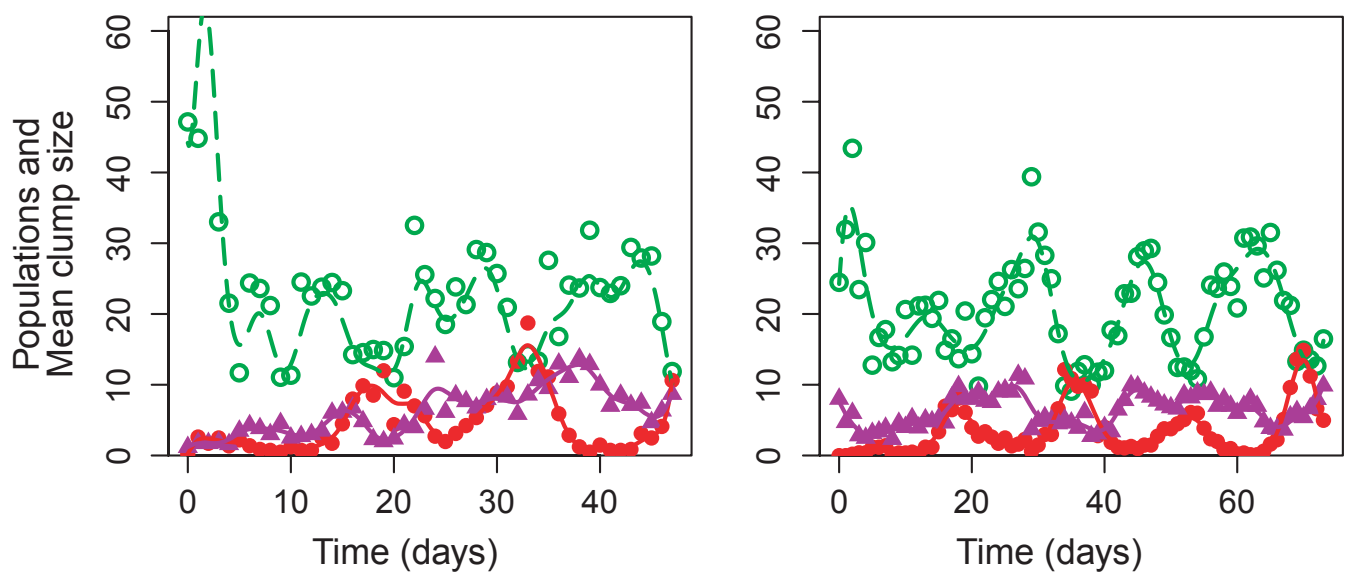

Figure 1 Population and mean trait dynamics (algal palmelloid clump size) of replicated chemostat experiments. Predator densities of the rotifer Brachionus (red lines, solid circles: females per millilitre), prey densities of the green alga Chlamydomonas (green dashed lines, open circles: $10^{4}$ cells $\mathrm{mL}^{-1}$ ) and the mean clump size of the prey population (purple line, triangles; data redrawn from Becks et al. 2010). The plotted curves are smooths of the data using cubic local polynomial regression with plugin bandwidth selection (Cabrera 2009). Vertical dotted lines in panel (A) and lower-case letters $b-i$ indicate the time points when algae were harvested for gene expression pattern analysis (Material and Methods). Numerical values adjacent to the dotted lines give the estimated percentage of edible algal cells at that time point (Figure 2).

inoculate the chemostat was the standard against which algae from later samples were compared (i.e. the 'grazed Chlamydomonas'). To obtain sufficient material for microarray analysis, c. $1-1.5 \mathrm{~L}$ algal samples were collected and filtered through a $10 \mu \mathrm{m}$ mesh to exclude rotifers and rotifer eggs. The algal samples of $c .100 \mathrm{~mL}$ of chemostat medium were concentrated by centrifugation and frozen. Samples were shock frozen in liquid nitrogen and transferred to $-80^{\circ} \mathrm{C}$.

\section{Molecular dynamics: microarray design}

We used customised microarrays (Agilent single glass slides formatted with eight $15 \mathrm{~K}$ arrays). Array probes (oligonucleotides) were taken from the design of Chlamy-Chip Version 2 (Eberhard et al. 2006). All annotated oligo-sequences of Chlamy-Chip Version 2 (c. 5000) were used (MapMan annotation for Chlamy-Chip Version 2 based on JGI3.1 Annotation; Thimm et al. 2004; May et al. 2009), plus 2500 sequences without annotation. Because the maximum possible probe length on Agilent CustomArrays is $60 \mathrm{bp}$, longer sequences were shortened by only the middle $60 \mathrm{bp}$. The Agilent $8 \times 15$ CustomArray combines eight arrays, each with space for 15802 probes, allowing us to duplicate all 7500 sequences. Sixteen arrays were hybridised and scanned resulting in four replicated hybridizations for each oligo-sequence for each time point (two replicated spots per array and two arrays with flipped dyes). For details on RNA extraction, cDNA synthesis and microarray data analysis see Supplementary Information and Table S1.

\section{RESULTS}

\section{Predator - prey - trait cycles}

In four replicate chemostats (Fig. 1), Brachionus and Chlamydomonas abundance exhibited evolutionary cycles (sensu Yoshida et al. 2007) with nearly antiphase oscillations of predator and prey abundance (predator maxima occurring just slightly before prey minima and vice-versa). This deviation from classical predator-prey cycles (in which predators lag prey by one-quarter cycle period) is expected theoretically when prey defence cycles as a result of evolution caused by selective prey mortality (Abrams 2000; Yoshida et al. 2003; Jones \& Ellner 2007). The prey evolves defences when predation is intense, and lose that defence (but gain competitive ability) when predators are scarce and prey are abundant. The maximum level of defence is predicted to occur between successive peaks in predator and prey density (Fig. 3a; Yoshida et al. 2003; Becks et al. 2010) clearly seen in Fig. 1A: when the level of defence (mean clump size) decreases because rotifer densities 
have recently been low, the rotifer population can increase, but when algal defence increases again because rotifer densities have been high, the rotifers decline. As predicted by the mathematical model for this system (Becks et al. 2010; Ellner \& Becks 2011; see Fig. 3a), the cycles in rotifer density lagged the algal cycles by slightly under half the cycle period ( $0.42 \pm 0.01$ periods, mean $\pm \mathrm{SE}$ across chemostats), whereas increases in defence (mean clump size) preceded increases in algal density $(0.19 \pm 0.04$ cycle periods $)$.

Similar predator-prey phase relations can also be produced by predator stage- or size-structure under certain conditions (de Roos \& Persson 2003). We therefore verified that algal defence evolution underlies these patterns by analysing the separate dynamics of edible and inedible prey (see Methods). With results from all chemostats pooled, the rotifer growth rate is best explained overall by the abundance of Chlamydomonas cells in clumps of eight or fewer cells (Fig. 2). We call this the edible fraction and larger clumps are called the inedible fraction. Microscope observations confirmed that Brachionus could not handle or consume the larger clumps (L. Becks and N. G. Hairston, Jr., pers. obs.).

The separate dynamics of the edible and inedible fractions of the algal population (Fig. 3c-f) are strikingly close to the predictions (Fig. 3b) of models that incorporate prey evolution (Ellner \& Becks 2011). Oscillations in the edible prey fraction (blue curves) lagged behind those of the inedible prey fraction (black curves) by $0.29 \pm 0.05$ (mean $\pm \mathrm{SE}$ ) cycle periods, whereas the predator populations (red curves) lagged the abundance of edible prey by approximately one-quarter period ( $0.24 \pm 0.04$ periods). Thus, the predators and the edible prey exhibited a standard predator-prey cycle. When all prey types are included, rotifer population and total algal prey (edible and inedible) exhibited antiphase evolutionary cycling (Figs 1 and 3a).

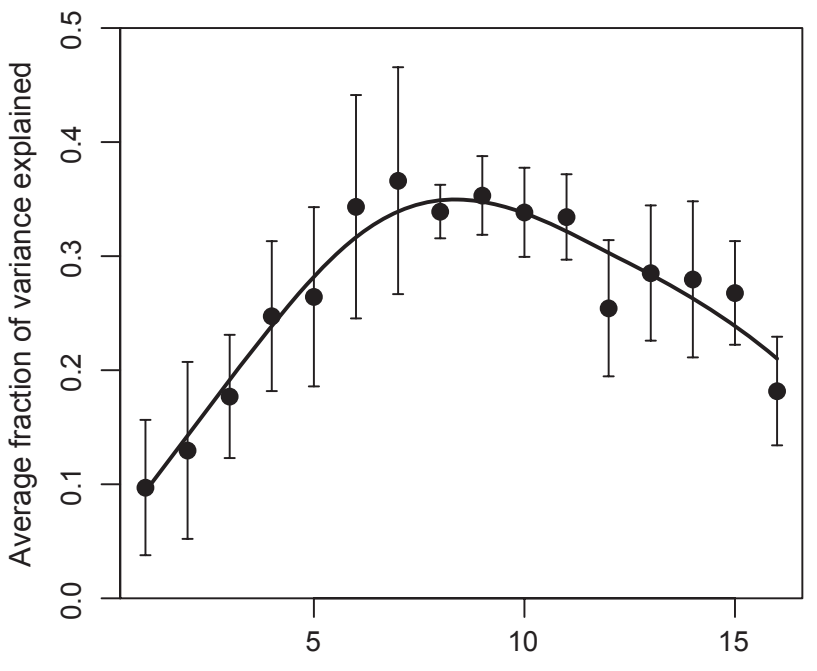

Maximum edible clump size

Figure 2 The relationship between the maximum size of algal clumps included in the 'edible' group, and the fraction of variance in rotifer per-capita growth rate explained by the abundance of these edible algae (the total number of algal cells in edible clumps). Dots are the mean of the $r^{2}$ values for the four replicate chemostats analysed separately, and the error bars show standard errors of the across-replicate variation. The solid line is a fitted spline regression (see Material and Methods), and the clump size at which the curve peaks is interpreted to be the maximum edible size.

\section{Ecological and evolutionary contributions to rotifer growth rate}

We used data on algal population abundance and changes in the fraction of edible algae to calculate the relative importance of ecological and evolutionary processes for rotifer population growth, using the 'Geber method' (Hairston et al. 2005). Rotifer population growth rate is expected to be an increasing, saturating function of prey density. At the same time, changes in prey edibility (i.e. clump size) also influence rotifer growth rate. The relative importance of these two effects can be partitioned through a two-way ANOVA so long as prey phenotype is highly heritable (Hairston et al. 2005; Ellner et al. 2011). Comparing the ecological and evolutionary effects day-by-day for each chemostat run shows that their relative importance fluctuated, often in opposite directions, but with a time lag (Fig. 4). This is expected, because increases in algal defence preceded increases in algal abundance by a quarter period (Fig. 1), as theory predicts (Jones \& Ellner 2007). Summing the daily effects of evolution and ecology for each chemostat run, the overall effect of evolution (changes in algal cell clumping) on rotifer growth rate was in all cases equal to or greater than the effect of ecological change (changes in algal density; the ratios of evolution : ecology effects are given in each panel in Fig. 4).

\section{Dynamics of gene expression}

For one of our four chemostats, we harvested algal samples for analysis of changes in the expression of 7500 candidate genes (see Material and Methods and Supplementary Information) at eight time points (Fig. 1A) encompassing two cycles of decrease and increase in cell clumping (time points $c-d-e$ and $f-g-h)$. Levels of algal gene expression were standardised against a sample taken from the clumpforming algal stock culture that was used to inoculate the chemostat at the start. Analysis of the resulting microarray data revealed between 4 and 95 genes that significantly $(P<0.05)$ changed in expression by at least two-fold between successive sample times.

For the dataset as a whole, we found that the total number of genes either up- or down-regulated between two consecutive time points (by at least two-fold at $P<0.05$ ), was positively related to the magnitude of the change (increase or decrease) in mean clump size [Pearson correlation coefficient $\rho=0.66$ between $\log _{2}$ (number of genes) and the absolute change in $\log _{2}$ (mean clump size), $P=0.03$; Fig. S2]. For example, of the seven sampling intervals in Fig. 1A, the three for which the change in clumping was smallest $(b-c, e-f, b-i$, Fig. 1A) had the fewest number of genes (20.7 on average) changing significantly in expression, whereas the four intervals with largest changes in clumping $(c-d, d-e, f-g, g-b)$ had more than three times this number of genes (72.8 on average) showing at least a two-fold change in expression.

To identify individual genes potentially involved in the formation and costs of cell clumps, we compared the extent of up- or downregulation of genes against changes in cell clumping between consecutive time points in the rotifer-algal dynamics. Across the full data set, we found no significant correlations (Pearson correlation coefficient, $\alpha<0.05$ after Benjamini-Hochberg correction for multiple comparisons) between the level of expression of particular Chlamydomonas genes and either the mean size of clumps, or the fraction of cells in the edible range of clump sizes $(\leq 8$ cells per clump; see Table S2; Fig. S3 for all genes that changed expression significantly between at least one pair of time points). Similarly, we 

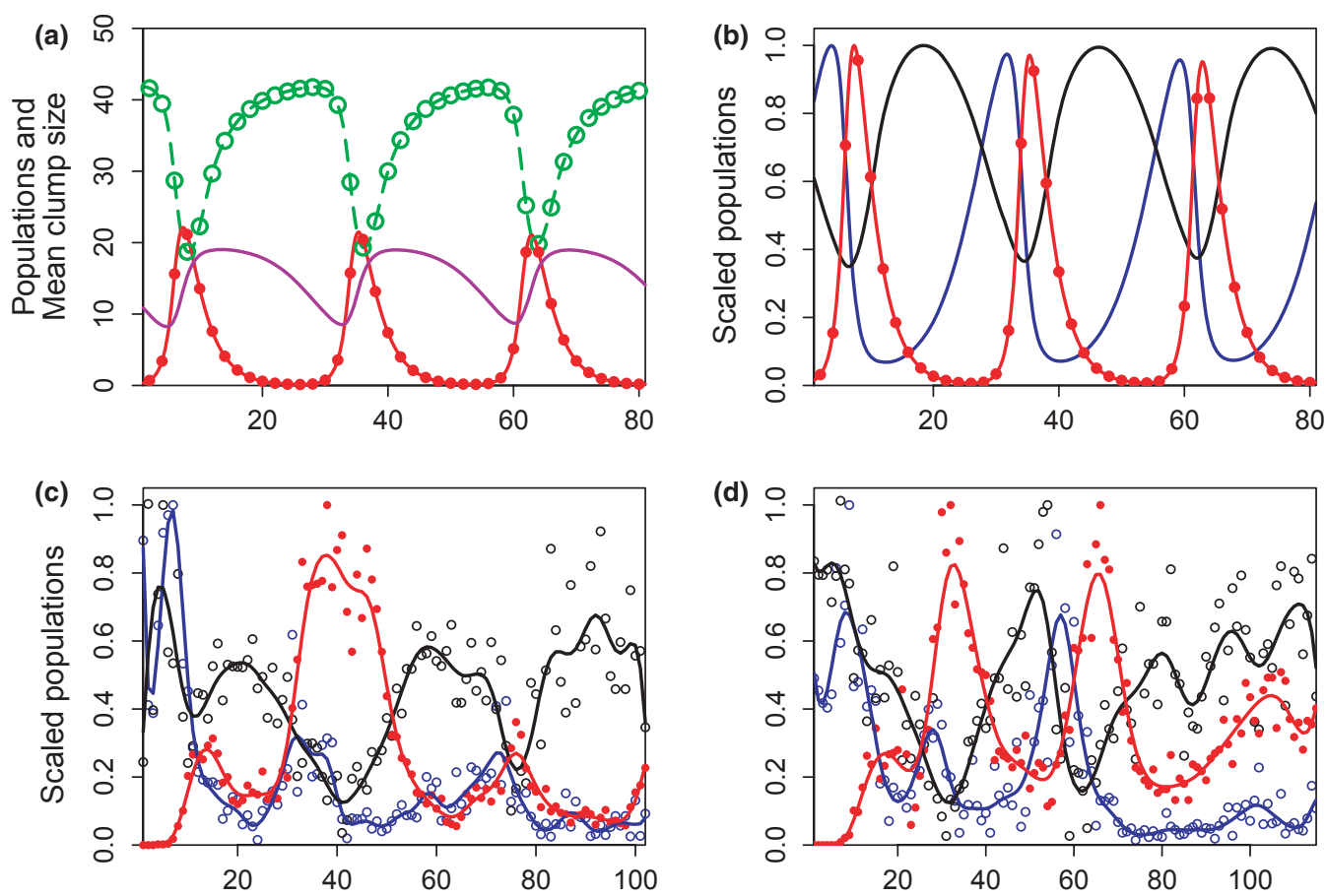

(d)
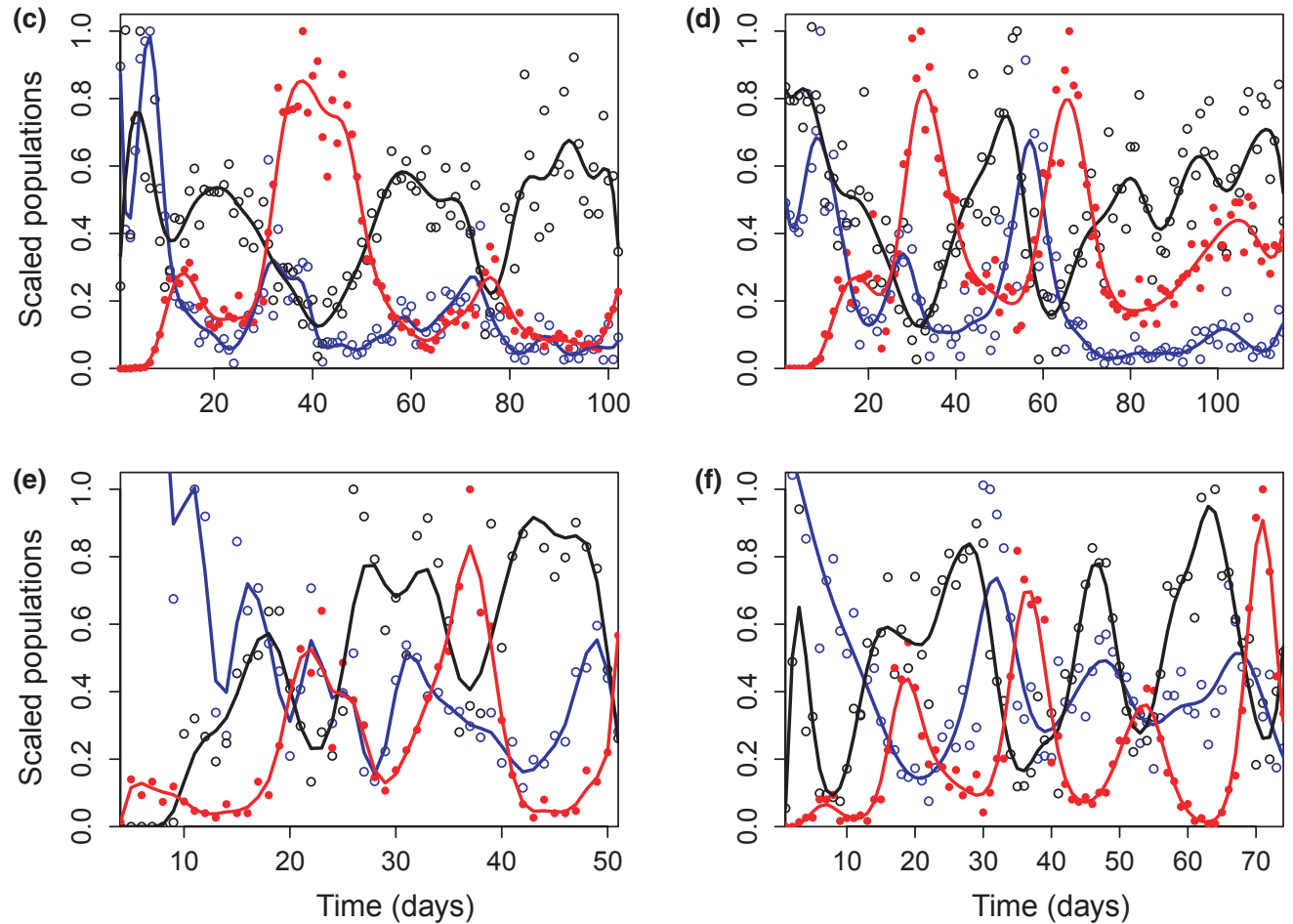

Figure 3 (a, b) Predicted dynamics (Becks et al. 2010) for system with predator, one edible and one wholly inedible prey clone: (a) total prey (green dashed line with open circles), predators (red with solid circles) and average level of prey defence (purple) and (b) density of predators (red with solid circles) plus edible (blue) and inedible prey (black). (c-f) Experimental results for rotifers (red), and edible (blue) and inedible (black, $\geq 8$ cells per clump, see Fig. 2) prey. Data smoothed using local polynomial regression with plugin bandwidth selection (Cabrera 2009). (b-f) Populations scaled relative to peak values.

found no genes with a significant overall difference in mean expression between the times of higher ( $d$ and $g$ ) vs. lower $(c, e, f$ and $h$ ) fraction of edible cells during the two cycles. However, when each of the two cycles of change in cell clumping are analysed separately, interesting patterns emerge in the expression of some categories of functional genes. We describe these responses elsewhere, after we explain why the two cycles need to be analysed separately.

There is consistency in the identity of genes up- or down-regulated within each of the two trait cycles seen in Figs $1 \mathrm{~A}$ and 3c. Time sequences $c-d-e$ and $f-g-h$ (i.e. those with the greatest changes in clumping) show the same eco-evolutionary trait dynamics: first a step-down in clumping ( $c-d$ and $f-g$ ) leading to increased rotifer density, and then a step-up in clumping $(d-e$ and $g-h)$ along with a decline in rotifer density. The microarray results (Fig. 5) show that during each cycle separately, genes up-regulated as clumping decreased tended to be down-regulated when clumping subsequently increased (Fig. 5A,B); similarly, those down-regulated when clumping decreased tended to be up-regulated when clumping subsequently increased, and genes that were neither up- or down-regulated during the decrease in clumping also did not change during the increase in clumping. Surprisingly, however, there was no statistical relationship between the changes in gene expression during the decrease in clumping in the first cycle, and the changes in expression during the corresponding decrease in clumping during the second cycle (Fig. 5C). Likewise there was no relationship between the gene regulation patterns during the increases in clumping in the two cycles (Fig. 5D).

Because the changes in gene expression during the two cycles were uncorrelated, we looked for genes that were markedly up or downregulated during each individual cycle. First, we used MapMan (Thimm et al. 2004) to determine functional categories that were significantly affected by changes in clumping (Table 1). Grouping genes according to likely common functional categories revealed trends in differential expression that were not obvious at the single gene level. Four functional groups of genes (plus a miscellaneous 

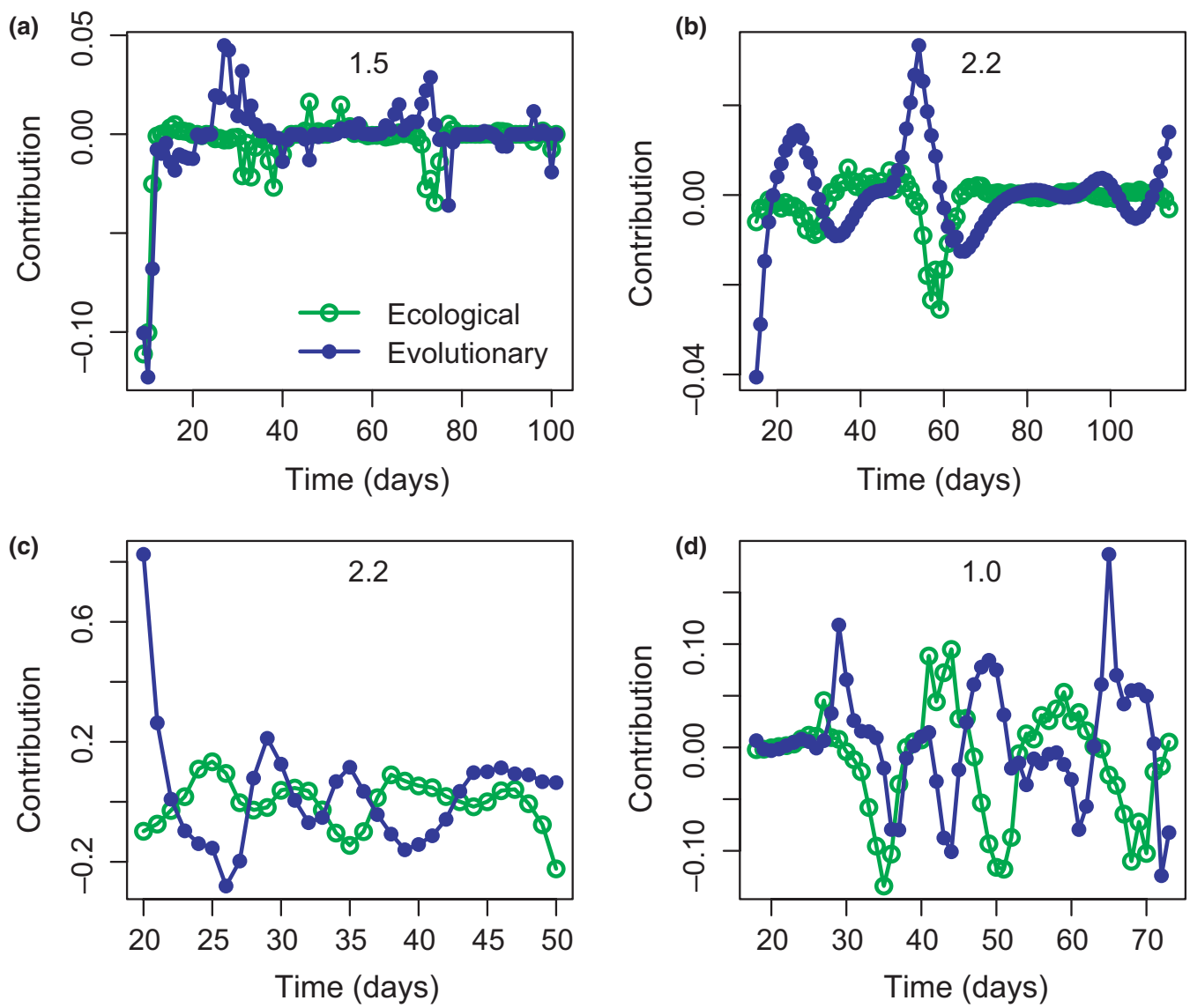

Figure 4 Contributions of ecological change (change in algal total abundance, green and open symbols) and evolutionary change (change in the defence trait as fraction of edible cells, blue and solid symbols) to rotifer population growth rate, calculated using the methods of Hairston et al. (2005), pp. 1119-1120). The layout of the four panels is the same as in Fig. 1. Numbers within the panels are the ratio between the total absolute contributions of evolutionary and ecological change over the time period plotted.

category of genes with unclear functional significance) changed in concert with cell clumping (i.e. either increased or decreased between two consecutive time points); genes involved in photosynthesis and protein metabolism were significantly differently expressed relative to all other functional groups when looking at changes in gene expression between consecutive time points. Genes involved in cell organization and cell cycle were significantly differently expressed for the second decrease $(f-g)$ and increase in clumping $(g-h)$, but not during the first cycle in clumping $(c-d-e)$. The cell wall category was only significantly affected during the first decrease in clumping $(c-d)$.

We then identified single genes that changed significantly in gene expression by at least two-fold between consecutive time points during a cycle for the functional groups identified in Table 1. There were 55 genes for the first cycle, 68 genes for the second cycle, and 45 genes in both cycles that were at least two-fold up or down-regulated (Table S2; Fig. S3 for all genes that changed expression significantly between at least one pair of time points; Table S3 for gene expression data for individual time points for the genes that show a significant two-fold change between consecutive time points). As was the case for gene expression as a whole, the direction of the expression for functional groups changed from the first to the second cycle for most genes. For photosynthesis-related genes, protein metabolism-related genes, and genes associated with the cell cycle and cell wall, those that were up-regulated in the first cycle as clumping declined $(c-d)$ and then down-regulated as clumping increased ( $d-e)$, subsequently reversed direction at the equivalent points in the second cycle (down-regulation with decreased clumping $f-g$; upregulation with increased clumping $g-h$; Fig. $5 \mathrm{E}$ ). For all three of these functional groups, most individual genes changed expression in both the first and second cycles (red lines), but again, the direction of change in regulation reversed from first to the second cycle (Fig. 5E). Cell wall and cycle genes are likely associated with the production of the clumping phenotype, because clumping in Chlamydomonas is the result of cells not fully disaggregating following cell division John et al. 2002). Most genes related to protein metabolism that showed a twofold expression change during the cycles are associated with nitrogen deprivation (Miller et al. 2010). As pointed out earlier, the clumping defence exacts a cost in population growth rate presumably because it decreases the availability of the limiting nutrient (nitrogen in our chemostats) to cells in the interior of clumps. It also seems likely that the marked changes in expression by genes involved in photosynthesis represent costs of defence that show up in primary metabolism, a phenomenon common in other studies of plant defence against herbivory (Kessler \& Heil 2011). As will be discussed elsewhere, because the environment in our chemostats is constant (except for the internal predator-prey dynamics that are the object of study), this consistent but counter-intuitive result from microarrays strongly suggests that gene regulation evolved from one control pathway to a different one between the two consecutive prey-trait cycles. 

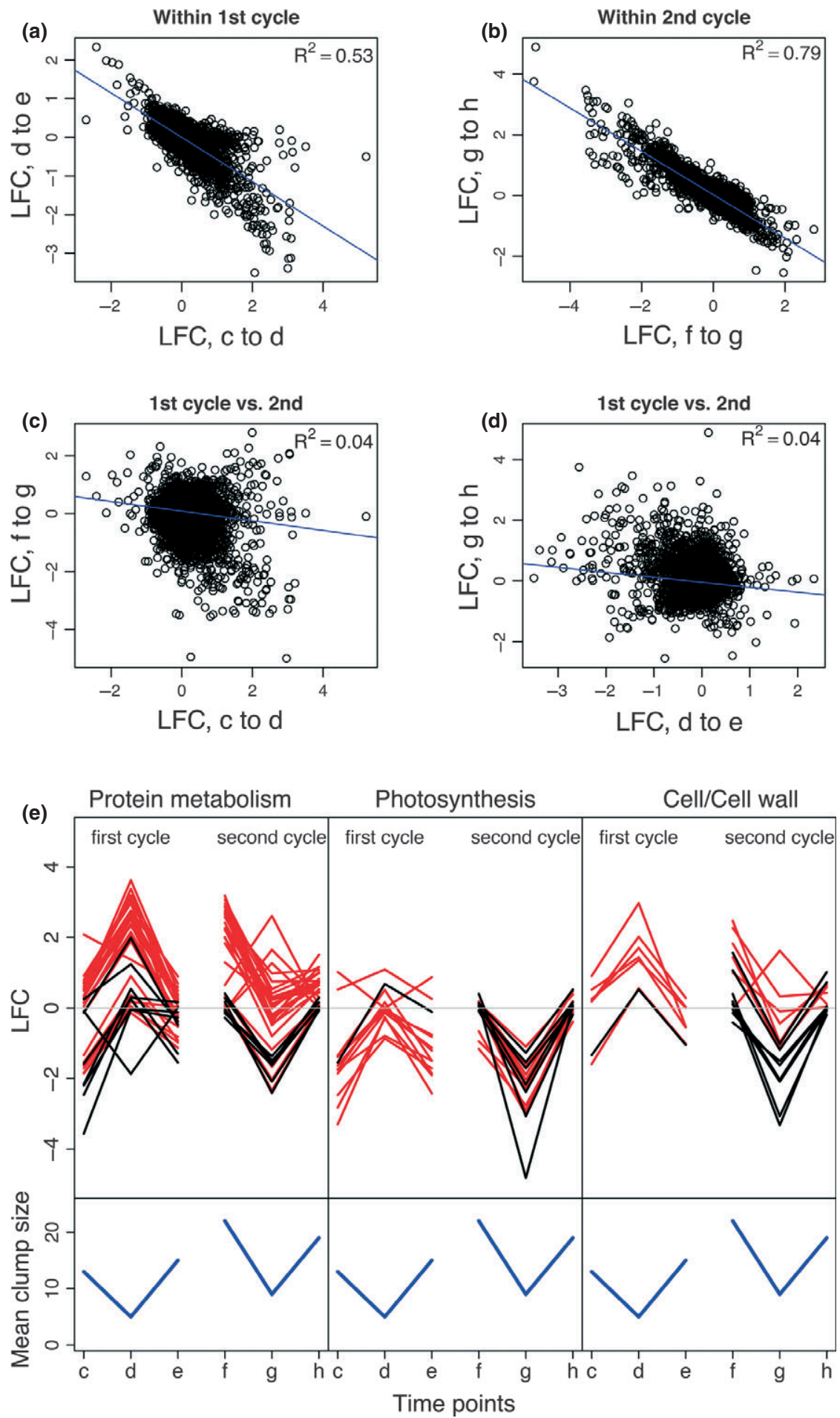

Figure 5 Gene expression during consecutive predator-prey cycles (Fig. 1A): changes during (A) the first (time points $c-d$ ) step-down in defence (clumping decrease) vs. those by the same genes during the subsequent step-up $(d-e)$ in defence. Log Fold Change (Log 2 LFC $=1$ is a two-fold change), (B) second step-up $(f-g)$ and step-down $(g-h)$, (C) first and second step-downs and (D) first and second step-ups. (E) Gene expression levels (log fold change relative to time point (a) for genes with significant two-fold change in both (red lines) or only one (black lines) of the cycles in mean clump size (blue lines). Functional groups of genes identified using MapMan (Table 1): gene IDs are listed in Table S2 and expression data in Table S3.

\section{DISCUSSION}

We have provided direct evidence for a complete eco-evolutionary feedback loop in which rapid prey evolution modifies an iconic ecological interaction, predator-prey cycling, in exactly the ways that theory predicted (Figs 1 and 3). Algae evolved to produce cell clumps too large ( $>8$ cells per clump; Fig. 2) for rotifers to consume effectively. Thus, algal evolution altered the food environment of the rotifers, with subsequent consequences for the environment of the algae when the predominance of defended algal genotypes caused the 
Table 1 Functional gene categories significantly affected by change the defence trait

\begin{tabular}{|c|c|c|c|c|}
\hline \multirow{2}{*}{$\begin{array}{l}\text { Functional } \\
\text { groups }\end{array}$} & Step-down 1 & Step-up 1 & Step-down 2 & Step-up 2 \\
\hline & $(c-d)$ & $(d-e)$ & $(f-g)$ & $(g-b)$ \\
\hline Photosynthesis & $<0.001$ & $<0.001$ & $<0.001$ & $<0.001$ \\
\hline Protein & $<0.001$ & $<0.001$ & $<0.001$ & $<0.001$ \\
\hline Cell cycle & - & - & $<0.001$ & $<0.001$ \\
\hline Cell wall & $<0.001$ & - & - & - \\
\hline Misc & $<0.001$ & $<0.001$ & $<0.001$ & $<0.001$ \\
\hline
\end{tabular}

Table entries are $P$-values for significantly different behaviour of a functional group, calculated based on Wilcoxon rank sum tests with Benjamini-Hochberg correction for multiple tests. MapMan (Thimm et al. 2004) was used to identify functional categories that exhibited significantly different behaviour in terms of expression profile compared with all other remaining functional groups (a total of 36 functional groups were considered, including c. $50 \%$ of the oligo-sequences on the array) for the gene expression between two consecutive time points of a cycle in clumping $(c-d, d-e, f-g, g-b)$. All functional groups that are significantly affected by changes in clumping are reported here.

rotifer population to decline. This allowed the algal population to increase, but also led to expression of the cost for defence - a lower population growth when competing for limiting nutrients (Becks et al. 2010). Because the clumped cells are connected in a mucilaginous matrix (dividing cells fail to separate; John et al. 2002), it is likely that clumped cells experience reduced diffusion of nitrogen (the limiting nutrient in our chemostats) to cell surfaces where it can be taken up. The resulting selection for competitive ability rather than defence led to the algal population becoming dominated by genotypes that occur as single cells or small clumps. The rotifers' food environment was then favourable, allowing the rotifer population to re-grow and initiate the cycle. Changes in the frequency of the defended prey genotype (i.e. evolution) played at least as important a role as changes in total prey density (i.e. ecology) in determining the growth of the rotifer population (Fig. 4).

This is, then, a complete eco-evolutionary feedback loop of the kind discussed by Fussmann et al. (2007), Kokko \& Lopez-Sepulcre (2007) and Schoener (2011), and meets the criteria defined by Post \& Palkovacs (2009): (1) the algal phenotype had a strong effect on its environment (rotifer population growth and subsequent rates of predation on the algae) and (2) the changed environment caused subsequent evolution of the algal population.

We also identified changes in algal gene expression that accompanied the eco-evolutionary dynamics. The patterns we observed in Chlamydomonas gene expression could have resulted from changes in alleles and allele frequencies (i.e. at the level of gene sequences), from regulatory changes of genes without change in underlying sequences, or both. Furthermore, because one regulatory gene may affect many other genes, individual genes may not be independent observations (e.g. Cooper et al. 2003). In addition, some genes involved in trait expression may not have been included on our microarray, or may have been missed by the RNA hybridization in our protocol.

The functional-genomic basis of the clumping phenotype proved to be complex in interesting ways. The significant relationship between the number of genes that were significantly up or down-regulated ( $\geq 2$-fold change) and the change in mean number of cells per clump shows that expression of many genes was linked to evolution of the defence trait. Either one or a few regulatory genes influenced expression at many loci, one of which controls cell clumping, or many genes contributed to producing the clumping phenotype (cf. Pigliucci et al. 1998). It is striking that there was no correspondence between the changes in gene expression during the first and second cycle of decrease and increase in cell clumping (Fig. 5A-D), so that two different suites of molecular mechanisms produced the same phenotypic change. This conclusion is supported by our comparison of the specific genes up- or down-regulated in the first cycle with the second cycle (Fig. 5E). Both a number of genes likely to be involved directly in cell clumping (i.e. cell wall and cycle genes), and a number known to respond to nitrogen deprivation responded significantly during both cycles. In each, however, the responses were in opposite directions for the two cycles. Because these dynamics were studied in a highly controlled laboratory environment, we have in essence a sequential common-garden experiment of two bouts of identical selection. The consistency of gene regulation patterns during decrease and increase in clumping within each trait cycle shows that the identified genes are indeed correlated with the clumping phenotype. However, the distinct or even opposite patterns of change in gene expression between the two cycles strongly suggests that there was evolution from one cycle to the next of the gene or genes regulating expression of the sequences on our microarrays. We have seen similarly rapid evolution in other experiments, where Chlamydomonas clones without any prior exposure to rotifers evolved the clumping trait within 10-30 days, after only one or two periods of high-rotifer predation (Becks et al. 2010). Our results are thus a caution that, even within a single narrowly confined population experiencing two nearly identical bouts of selection and evolutionary response, there can be multiple molecular mechanisms underlying a particular adaptive change in phenotype. However, detailed gene expression studies including more replicated populations and more time points during cycles will be needed to fully understand the evolution of clumping.

Although there are examples of self-contained eco-evolutionary feedback loops that operate entirely within a single species where its own density influences selection for characters that affect density (e.g. Hanski 2011), and those were rare genotypes have a selective advantage simply because they are rare (e.g. Sinervo et al. 2000), our interest here is in feedbacks where the effects of evolution in one species influence ecological processes in another and so alter selection on the first. There have been, until now, few if any examples of complete eco-evolutionary feedback loops of this latter kind (Post \& Palkovacs 2009; Schoener 2011). One possible explanation is that their occurrence requires tightly coupled interactions among a few species and their heritable traits, conditions that we created in our microcosms. These conditions may not be typical of natural communities, where many species affect each other with varying interaction strength (e.g. Paine 1992), via pathways of varying complexity (Wootton 1994), with differing amounts of spatial structure and dispersal (Leibold et al. 2004), and with differing degrees of influence from the physical environment (Strong 1986). Nevertheless, the complexities of natural ecosystems do not necessarily preclude eco-evolutionary dynamics, so it is worth asking how common and under what conditions eco-evolutionary feedback loops are expected (Ellner et al. 2011; Schoener 2011).

One place to begin looking for strong eco-evolutionary feedbacks in nature may be host-parasite or host-pathogen interactions, particularly host-specific parasites or pathogens whose ecological and evolutionary interactions are tightly coupled with their host's. One example can be found in freshwater crustaceans of the genus Daphnia and their microbial parasites. Daphnia populations in many lakes in 
North America and Europe are attacked by virulent pathogens, but recover when resistant genotypes evolve, often within a single season (Wolinska et al. 2008; Duffy et al. 2009; Penczykowski et al. 2011), which for these animals can represent dozens of generations. As in our predator-prey system, an eco-evolutionary feedback loop can result if evolution of increased host resistance leads to decreased pathogen prevalence, so that costs of resistance (e.g. reduced feeding and juvenile growth rates in D. dentifera; Hall et al. 2010) generate selection on the host for competitive ability rather than pathogen resistance. A different kind of eco-evolutionary feedback loop can occur if pathogen virulence evolves in response to the evolution of resistance by the host (e.g. Decaestecker et al. 2007; Wolinska et al. 2008). The recent genome sequencing (Colbourne et al. 2011) of the new environmental genomics model organism Daphnia pulex should create opportunities to study the links between genetic and ecological dynamics in an ecologically significant species under natural conditions.

\section{ACKNOWLEDGEMENTS}

We thank Matthew Cortese, Theodore Hermann, Alicia Lande, Nancy Pyne, Derek West, Lynn Wilking, and Stephanie Yager for sampling and maintaining of chemostats, Colleen Kearns for advice and assistance, and Giles Hooker and Haim Bar for advice on microarray data analysis. We are grateful to Jeffey Pleiss and his group for advice and support on the microarray experiments and to David Stern for discussions about the results. This research was funded by grants from the Andrew W. Mellon Foundation to NGH Jr. and SPE, and from the James S. McDonnell Foundation to SPE, NGH, LEJ and G. Fussmann. The funders had no role in study design, data collection and analysis, decision to publish, or preparation of the manuscript.

\section{COMPETING INTERESTS}

The authors have declared that no competing interests exist.

\section{AUTHOR CONTRIBUTIONS}

LB, SPE, LEJ and NGH Jr. conceived and designed the study; LB performed experiments; SPE and LB analysed microarray data. All authors analysed population data, discussed the results and participated in writing the paper.

\section{REFERENCES}

Abrams, P.A. (2000). The evolution of predator-prey interactions: theory and evidence. Annu. Rev. Ecol. Syst., 31, 79-105.

Barrett, R.D., MacLean, R.C. \& Bell, G. (2005). Experimental evolution of Pseudomonas fluorescens in simple and complex environments. Am. Nat., 166, 470-480.

Becks, L., Ellner, S.P., Jones, J.L. \& Hairston, N.G. Jr. (2010). Reduction of adaptive genetic diversity radically alters eco-evolutionary community dynamics. Ecol. Lett., 13, 989-997.

Bell, M.A. (2009). Microevolution. In: The Princeton Guide to Ecology (ed. Levin, S.A.). Princeton University Press, Princeton and Oxford, pp. 126-133.

Cabrera, J.L.O. (2009). locpol: kernel local polynomial regression. R package version 0.4-0. Available at: http://CRAN.R-project.org/package=locpol. Last accessed 1 January 2012.

Colbourne, J.K., Pfrender, M.E., Gilbert, D., Thomas, W.K., Tucker, A., Oakley, T.H. et al. (2011). The ecoresponsive genome of Daphnia pulex. Science, 331, 555-561.

Cooper, B., Clarke, J.D., Budworth, P., Kreps, J., Hutchison, D., Park, S. et al. (2003). A network of rice genes associated with stress response and seed development. Proc. Natl. Acad. Sci. USA, 100, 4945-4950.
Decaestecker, E., Gaba, S., Raeymaekers, J.A.M., Stoks, R., Van Kerckhoven, L., Ebert, D. et al. (2007). Host-parasite 'Red Queen' dynamics archived in pond sediment. Nature, 450, 870-873.

Duffy, M.A., Hall, S.R., Caceres, C.E. \& Ives, A.R. (2009). Rapid evolution, seasonality, and the termination of parasite epidemics. Ecology, 90, 1441-1448.

Eberhard, S., Jain, M., Im, C.S., Pollock, S., Shrager, J., Lin, Y.A. et al. (2006). Generation of an oligonucleotide array for analysis of gene expression in Chlamydomonas reinhardtii. Curr. Genet., 49, 106-124.

Ellner, S.P. \& Becks, L. (2011). Rapid prey evolution and the dynamics of two-predator food webs. Theor. Ecol., 4, 133-152.

Ellner, S.P., Geber, M.A. \& Hairston, N.G. Jr. (2011). Does rapid evolution matter? Measuring the rate of contemporary evolution and its impacts on ecological dynamics Ecol. Lett., 14, 603-614.

Fussmann, G.F., Ellner, S.P., Shertzer, K.W. \& Hairston, N.G. Jr. (2000). Crossing the Hopf bifurcation in a live predator-prey system. Science, 290, 1358-1360.

Fussmann, G.F., Loreau, M. \& Abrams, P.A. (2007). Eco-evolutionary dynamics of communities and ecosystems. Funct. Ecol., 21, 465-477.

Gibson, G. (2002). Microarrays in ecology and evolution: a preview. Mol. Ecol., 11, $17-24$.

Hairston, N.G. Jr., Ellner, S.P., Geber, M.A., Yoshida, T. \& Fox, J.A. (2005). Rapid evolution and the convergence of ecological and evolutionary time. Ecol. Lett., 8, $1114-1127$.

Hall, S.R., Becker, C.R., Duffy, M.A. \& Caceres, C.E. (2010). Variation in resource acquisition and use among host clones creates key epidemiological trade-offs. Am. Nat., 176, 557-565.

Hanski, I.A. (2011). Eco-evolutionary spatial dynamics in the Glanville fritillary butterfly. Proc. Natl. Acad. Sci. USA, 108, 14397-14404.

Hendry, A.P. \& Kinnison, M.T. (1999). Perspective: the pace of modern life: measuring rates of contemporary microevolution. Evolution, 53, 1637-1653.

John, D.M., Whitton, B.A. \& Brook, A.J. (2002). The Freshwater Algal Flora of the British Isles: An Identification Guide to Freshwater and Terrestrial Algae. Cambridge University Press, Cambridge.

Jones, L.E. \& Ellner, S.P. (2007). Effects of rapid prey evolution on predator-prey cycles. J. Math. Biol., 55, 541-573.

Kessler, A. \& Heil, M. (2011). The multiple faces of indirect defences and their agents of natural selection. Funct. Ecol., 25, 348-357.

Kokko, H. \& Lopez-Sepulcre, A. (2007). The ecogenetic link between demography and evolution: can we bridge the gap between theory and data? Ecol. Lett., 10, 773-782.

Leibold, M.A., Holyoak, M., Mouquet, N., Amarasekare, P., Chase, J.M., Hoopes, M.F. et al. (2004). The metacommunity concept: a framework for multi-scale community ecology. Ecol. Lett., 7, 601-613.

Lenski, R.E., Rose, M.R., Simpson, S.C. \& Tadler, S.C. (1991). Long-term experimental evolution in Escherichia coli. 1 Adaptation and divergence during 2000 generations. Am. Nat., 138, 1315-1341.

Levin, S.A. (1972). Mathematical analysis of genetic feedback mechanism. Am. Nat., 106, 145-164.

Lopez, D., Casero, D., Cokus, S.J., Merchant, S.S. \& Pellegrini, M. (2011). Algal functional annotation tool: a web-based analysis suite to functionally interpret large gene lists using integrated annotation and expression data. BMC Bioinformatics, 12, 1-10.

Lürling, M. \& Beekman, W. (2006). Palmelloids formation in Chlamydomonas reinhardtii: defence against rotifer predators? Ann. Limnol. - Int. J. Lim., 42, 65-72.

May, P., Christian, J.-O., Kempa, S. \& Walther, D. (2009). ChlamyCyc: an integrative systems biology database and web-portal for Chlamydomonas reinhardtii. BMC Genomics, 10, 209.

Merchant, S.S., Prochnik, S.E., Vallon, O., Harris, E.H., Karpowicz, S.J., Witman, G.B. et al. (2007). The Chlamydomonas genome reveals the evolution of key animal and plant functions. Science, 318, 245-251.

Meyer, J.R., Ellner, S.P., Hairston, N.G. Jr., Jones, L.E. \& Yoshida, T. (2006). Prey evolution on the time scale of predator-prey dynamics revealed by allele-specific quantitative PCR. Proc. Natl. Acad. Sci. USA, 103, 10690-10695.

Miller, R., Wu, G.X., Deshpande, R.R., Vieler, A., Gartner, K., Li, X.B. et al. (2010). Changes in transcript abundance in Chlamydomonas reinhardtii following nitrogen deprivation predict diversion of metabolism. Plant Physiol., 154,1737-1752.

Paine, R.T. (1992). Food-web analysis through field measurement of per-capita interaction strength. Nature, 355, 73-75. 
Pelletier, F., Garant, D. \& Hendry, A.P. (2009). Eco-evolutionary dynamics: an introduction. Phil. Trans. R. Soc. B, 364, 1483-1489.

Penczykowski, R.M., Forde, S.E. \& Duffy, M.A. (2011). Rapid evolution as a possible constraint on emerging infectious diseases. Freshwater Biol., 56, 689-704.

Pigliucci, M., Tyler, G.A. \& Schlichting, C.D. (1998). Mutational effects on constraints on character evolution and phenotypic plasticity in Arabidopsis thaliana. J. Genet., 77, 95-103.

Post, D.M. \& Palkovacs, E.P. (2009). Eco-evolutionary feedbacks in community and ecosystem ecology: interactions between the ecological theatre and the evolutionary play. Phil. Tran. R. Soc. B, 364, 1629-1640.

R Development Core Team (2009). R: a language and environment for statistical computing. R Foundation for Statistical Computing, Vienna, Austria. ISBN 3900051-07-0. Available at: http://www.R-project.org. Last accessed 1 November 2011.

de Roos, A.M. \& Persson, L. (2003). Competition in size-structured populations: mechanisms inducing cohort formation and population cycles. Theor. Pop. Biol., 63, 1-16.

Schoener, T.W. (2011). The newest synthesis: understanding the interplay of evolutionary and ecological dynamics. Science, 331, 426-429.

Siepielski, A.M., DiBattista, J.D. \& Carlson, S.M. (2009). It's about time: the temporal dynamics of phenotypic selection in the wild. Ecol. Lett., 12, 1261-1276.

Sinervo, B., Svensson, E. \& Comendant, T. (2000). Density cycles and an offspring quantity and quality game driven by natural selection. Nature, 406, 985-988.

Strong, D.R. (1986). Density-vague population change. Trends Ecol. Evol., 1, 39-42.

Thimm, O., Blasing, O., Gibon, Y., Nagel, A., Meyer, S., Kruger, P. et al. (2004). MAPMAN: a user-driven tool to display genomics data sets onto diagrams of metabolic pathways and other biological processes. Plant J., 37, 914-939.

Wolinska, J., Lively, C.M. \& Spaak, P. (2008). Parasites in hybridizing communities: the Red Queen again? Trends Parasitol., 24, 121-126.

Wood, S.N. (2000). Modelling and smoothing parameter estimation with multiple quadratic penalties. J. Roy. Stat. Soc. B, 62, 413-428.
Wootton, J.T. (1994). Putting the pieces together - testing the independence of interactions among organisms. Ecology, 75, 1544-1551.

Yoshida, T., Jones, L.E., Ellner, S.P., Fussmann, G.F. \& Hairston, N.G. Jr. (2003). Rapid evolution drives ecological dynamics in a predator-prey system. Nature, 424, 303-306.

Yoshida, T., Hairston, N.G. Jr. \& Ellner, S.P. (2004). Evolutionary trade-off between defence against grazing and competitive ability in a simple unicellular alga, Chlorella vulgaris. Proc. R. Soc. B, 271, 1947-1953.

Yoshida, T., Ellner, S.P., Jones, L.E., Bohannan, B.J.M., Lenski, R.E. \& Hairston, N.G. Jr. (2007). Cryptic population dynamics: rapid evolution masks trophic interactions. PLoS Biol., 5, 1868-1879.

\section{SUPPORTING INFORMATION}

Additional Supporting Information may be downloaded via the online version of this article at Wiley Online Library (www.ecologyletters.com).

As a service to our authors and readers, this journal provides supporting information supplied by the authors. Such materials are peer-reviewed and may be re-organized for online delivery, but are not copy-edited or typeset. Technical support issues arising from supporting information (other than missing files) should be addressed to the authors.

Editor, Colleen Webb

Manuscript received 23 December 2011

First decision made 3 February 2012

Manuscript accepted 16 February 2012 